\title{
Endovascular Treatment of Ischemic Stroke, Are we Far from the Evidence?
}

\author{
Gustavo J. Rodriguez ${ }^{1 *}$ and Alberto Maud ${ }^{2}$ \\ ${ }^{1}$ Gustavo J. Rodriguez, Neurology Department, Paul L. Foster School of Medicine, Texas Tech University Health Sciences Center, USA \\ ${ }^{2}$ Alberto Maud, Neurology and Radiology Departments, Paul L. Foster School of Medicine, Texas Tech University Health Sciences Center, USA
}

Received: November 06, 2013; Accepted: November 09, 2013; Published: November 12, 2013

"Corresponding author: Gustavo J. Rodriguez, Neurology Department, Radiology Department, Texas Tech University Health Sciences Center Paul L. Foster School of Medicine, USA, Tel: 915-545-6844;Fax: 915-545-6705; E-mail: gustavo.j.rodriguez@ttuhsc.edu

\section{Editorial}

As the population ages the incidence of stroke is expected to rise. Healthcare costs related to stroke including those costs associated to disability are projected to increase and will be unbearable. Despite the advances in the last two decades in the treatment of acute ischemic stroke and the approval of intravenous alteplase (rt-PA) by the FDA, around $50 \%$ of the patients still die or become dependent after ischemic stroke.

In an attempt to further improve the stroke outcomes, other treatment modalities in ischemic stroke were explored. The endovascular approach has theoretical advantages which include the use of mechanical disruption/embolectomy devices as facilitators for recanalization and/or the need of lower dosages of thrombolytic agents, locally infused within the thrombus with less systemic effects. The promising results of endovascular thrombolysis in The Prolyse in Acute Cerebral Thromboembolism (PROACT) II opened the door for further endovascular attempts of pharmacological and mechanical recanalization. The study compared prourokinase infused within 6 hours of symptom onset with placebo, for middle cerebral artery occlusions [1]. The favorable outcomes reported $(15 \%$ absolute increase in the probability of good neurological function modified Rankin Score $(\mathrm{mRS}) \leq 2$ and a $66 \%$ recanalization rate in the treatment group compared to placebo), have served as the historical control for the initial mechanical embolectomy devices and to the consideration of the endovascular treatment in the middle cerebral artery occlusion within 6 hours, as Class I evidence by the American Stroke Association in patients otherwise not eligible for intravenous rt-PA or those in whom is contraindicated. After the approval of the intravenous rt-PA for ischemic stroke, however no study has shown superiority of the endovascular treatments alone or in combination with intravenous rt-PA when compared to intravenous rt-PA alone, but has the comparison been fair?

There has never been a head to head comparison of IV rt-PA vs. endovascular treatment within a 3 hour window that included patients eligible for rt-PA. There are at least two important limitations to such "fair" comparison. First, such design will be considered unethical at this time when t-PA is the standard of care; thus any study design will have to include patients not eligible for rt-PA. Second, time delay in endovascular treatments is another important limiting factor that makes a fair comparison of these two modalities not feasible. However, it is important to highlight the importance of time. In the Interventional Management of Stroke (IMS) I and II trials, longer time to recanalization decreased the probability of favorable outcome such that recanalization provided no additional clinical benefit beyond $350 \mathrm{~min}$ from symptom onset [2]. This evidence and the experience in cardiology with the goal of percutaneous coronary intervention within 90 minutes from symptom onset [3], would suggest that an expedited approach to endovascular treatment of ischemic stroke is sorely needed but the recent negative trials of endovascular treatment make it hard to imagine a scenario in which any hospital system would invest in such processes yet the question is, should we try it?

Recently three randomized controlled trials showed negative and unexpected results, the criticism lies on the fact that newer devices were not used and endovascular treatments had major delays. These results lead to concerns about the efficacy of endovascular treatments within the medical community and now healthcare payers have threatened with denials of payments for endovascular treatments.

The Interventional Management of Stroke (IMS) III trial, was a randomized trial in which patients receiving intravenous rt-PA within $3 \mathrm{~h}$ were randomized to receive endovascular treatment or no additional treatment. The trial was discontinued after 656 participants had undergone randomization because of futility, however no harm was observed in the treatment group. Interestingly there was a trend towards better outcome in the treatment group for those treated within $2 \mathrm{~h}$ and those with time from intravenous rt-PA to groin puncture of less than 90 min [4].

The SYNTHESIS Expansion trial was a randomized trial 
in which patients within $4.5 \mathrm{~h}$ after onset were randomized to endovascular treatment, or intravenous rt-PA. No difference was observed in the primary outcome. There was a trend towards better outcome in the intravenous rt-PA group for patients older than 67 years and those with the National Institutes of Health Stroke Scale (NIHSS) score of $<11$ [5].

The (Magnetic Resonance and Recanalization of Stroke Clots Using Embolectomy) MR RESCUE trial was a randomized trial in which patients with ischemic stroke with large-vessel, anterior-circulation occlusion within $8 \mathrm{~h}$ after symptom onset to either endovascular treatment or standard of care, including intravenous rt-PA for eligible patients. Patients were stratified by the presence of a favorable penumbral pattern or not prior to randomization on pretreatment computed tomography (CT) or magnetic resonance imaging (MRI) of the brain. Patients with penumbral pattern had smaller infarct volumes and better outcomes regardless of the treatment modality [6].

Despite recent negative results much we have learnt regarding the endovascular treatment of ischemic stroke. More carefully study designs addressing the when and how to treat are needed. Expedited endovascular treatments with similar intravenous rtPA time goals and the use of the latest generations of mechanical embolectomy devices in centers with the appropriate expertise will soon lead to the so desired evidence.

\section{References}

1. Furlan, A., Higashida, R., Wechsler, L., et al. (1999). Intraarterial prourokinase for acute ischemic stroke. The PROACT II study: a randomized controlled trial. Prolyse in Acute Cerebral Thromboembolism. JAMA, 282, 2003-2011.

2. Khatri, P., Abruzzo, T., Yeatts, S. D., Nichols, C., Broderick, J. P., Tomsick, T. A., et al. (2009). Good clinical outcome after ischemic stroke with successful revascularization is time-dependent. Neurology, 73, 10661072.

3. Moscucci, M., Eagle, K. A. (2006). Door-to-balloon time in primary percutaneous coronary intervention: is the 90 -minute gold standard an unreachable chimera? Circulation, 113, 1048-1050.

4. Broderick, J. P., Palesch, Y. Y., Demchuk, A. M., Yeatts, S. D., Khatri, P., Hill, M. D., et al. (2013). Endovascular therapy after intravenous t-PA versus t-PA alone for stroke. The New England journal of medicine, 368, 893-903.

5. Ciccone, A., Valvassori, L., Nichelatti, M., Sgoifo, A., Ponzio, M., Sterzi, R., et al. (2013). Investigators SE. Endovascular treatment for acute ischemic stroke. $N$ Engl J Med, 368, 904-913.

6. Kidwell, C. S., Jahan, R., Gornbein, J., Alger, J. R., Nenov, V., Ajani, Z., et al. (2013). A trial of imaging selection and endovascular treatment for ischemic stroke. N Engl J Med 368, 914-923. http://www.ncbi.nlm. nih.gov/pubmed/10591382 